\title{
OPEN Early life dietary intervention in dairy calves results in a long-term reduction in methane emissions
}

\author{
S. J. Meale ${ }^{1,2}$, M. Popova ${ }^{1}$, C. Saro ${ }^{1,3}$, C. Martin ${ }^{1}$, A. Bernard ${ }^{1}$, M. Lagree ${ }^{4}$, D. R. Yáñez-Ruiz ${ }^{5}$, \\ H. Boudra ${ }^{1}$, S. Duval ${ }^{6}$ \& D. P. Morgavi ${ }^{1 凶}$
}

Recent evidence suggests that changes in microbial colonization of the rumen prior to weaning may imprint the rumen microbiome and impact phenotypes later in life. We investigated how dietary manipulation from birth influences growth, methane production, and gastrointestinal microbial ecology. At birth, 18 female Holstein and Montbéliarde calves were randomly assigned to either treatment or control (CONT). Treatment was 3-nitrooxypropanol (3-NOP), an investigational antimethanogenic compound that was administered daily from birth until three weeks post-weaning (week 14). Samples of rumen fluid and faecal content were collected at weeks 1, 4, 11, 14, 23, and 60 of life. Calves were tested for methane emissions using the GreenFeed system during the postweaning period (week 11-23 and week 56-60 of life). Calf physiological parameters (BW, ADG and individual VFA) were similar across groups throughout the trial. Treated calves showed a persistent reduction in methane emissions $\left(\mathrm{g} \mathrm{CH}_{4} / \mathrm{d}\right)$ throughout the post-weaning period up to at least 1 year of life, despite treatment ceasing three weeks post-weaning. Similarly, despite variability in the abundance of individual taxa across weeks, the rumen bacterial, archaeal and fungal structure differed between CONT and 3-NOP calves across all weeks, as visualised using sparse-PLS-DA. Similar separation was also observed in the faecal bacterial community. Interestingly, despite modest modifications to the abundance of rumen microbes, the reductive effect of 3-NOP on methane production persisted following cessation of the treatment period, perhaps indicating a differentiation of the ruminal microbial ecosystem or a host response triggered by the treatment in the early development phase.

Due to rising environmental concerns over methane emissions from ruminant production systems, many studies have attempted to target the ruminal microbiota, specifically methanogens, considered responsible. Not only does methane from agriculture account for $14 \%$ of total anthropogenic emissions ${ }^{1}$, but it also represents a loss of energy from the animal ${ }^{2}$. However, manipulating the mature ruminal microbiome in favour of reduced greenhouse gas emissions or increased production efficiency has proven challenging. Even following the application of antibiotics or complete rumen content exchanges, the microbiome inevitably shifts back to its pre-intervention state $^{3}$. Recent characterisations of the gastrointestinal microbiome from birth have outlined the sequential establishment of microbial domains ${ }^{4-6}$. This may provide a small window for effective manipulation of methanogens, as ruminal archaea reach levels similar to that of an adult by $\sim 14 \mathrm{~d}$ of life ${ }^{7,8}$. Abecia et al. ${ }^{9}$ showed the effective manipulation of the microbiome in goats, when the doe was treated in addition to the offspring until three months post weaning. Although the effect did not persist entirely 4 months after, we considered that interventions which target both microbial establishment, and the weaning transition period, may have the potential to imprint the microbiome for reduced methane emissions persisting later into life.

\footnotetext{
${ }^{1}$ INRAE, UMR Herbivores, VetAgro Sup, Université Clermont Auvergne, 63122 Saint-Genès Champanelle, France. ${ }^{2}$ School of Agriculture and Food Sciences, The University of Queensland, Gatton Campus, Gatton, QLD 4343, Australia. ${ }^{3}$ Departamento de Producción Animal, Universidad de León, Campus Vegazana s/n, 24071 León, Spain. ${ }^{4}$ Plateforme d'Exploration du Métabolisme, MetaboHUB Clermont, Université Clermont Auvergne, 63000 Clermont-Ferrand, France. ${ }^{5}$ Estación Experimental del Zaidín (CSIC), Profesor Albareda 1, 18008 Granada, Spain. ${ }^{6}$ Research Centre for Animal Nutrition and Health, DSM Nutritional Products, BP 170, 68305 Saint-Louis Cedex, France. ${ }^{\circledR}$ email: Diego.morgavi@inrae.fr
} 
Many studies have examined the use of dietary interventions to modulate the rumen microbiome or an animal's productive capacity with varying success. For example, the addition of live yeast or certain probiotics can increase feed efficiency via oxygen scavenging ${ }^{10,11}$, whereas, tannins form phenol-dietary protein complexes to improve the flow of digestible bypass protein ${ }^{12,13}$. Strategies to alter the microbiome in favour of reduced methanogenesis include saponins $s^{14,15}$, tannins, nitrates and unsaturated fats ${ }^{16-19}$, which exhibit an antiprotozoal effect. However, effects of these interventions have largely failed to persist beyond treatment application periods.

Here we considered the methane inhibitor, 3-nitrooxypropanol (3-NOP), which specifically targets methylcoenzyme $\mathrm{M}$ reductase (MCR), inhibiting catalysis of methane formation during rumen fermentation ${ }^{20}$. In adult ruminants, 3 -NOP has effectively inhibited methane by up to $30 \%$, and shown resistance to ruminal adaptation with effects persisting for up to 16 weeks with continuous treatment ${ }^{21}$. However, in a study by Romero-Perez et al. ${ }^{22}$ the effects diminished after treatment ceased, indicating that the inhibiting effects on methane are likely dependent on continuous supplementation of 3-NOP. Importantly, no negative effects have been reported, while a few studies have reported minor improvements in production that could be due to energy being redirected away from methanogenesis ${ }^{23,24}$. As such, we examined 3-NOP as a candidate early-life dietary intervention to modulate microbial establishment in the gastrointestinal tract of dairy calves. Further, we examined the effects of 3-NOP on methane production in post-weaned calves during treatment, and for up to one year post treatment to determine its ability to favour reduced methane production.

\section{Materials and methods}

The experiment was conducted at the INRAE dairy research farm (La boire, Marcenat, https://doi.org/10.15454 /1.5572318050509348E12). Procedures were evaluated and approved by the French Ministry of Education and Research (APAFIS \#4062-2015043014541577 v5), and carried out in accordance with French and European guidelines and regulations for animal experimentation. Information provided in the manuscript complies with the essential recommendations for reporting of the ARRIVE guidelines.

Animals, diets and experimental design. Eighteen female Holstein $(n=12)$, Montbéliarde $(n=4)$ and Holstein $x$ Montbéliarde $(\mathrm{n}=2)$ calves $(42.07 \pm 3.85 \mathrm{~kg}$ birth weight $)$ were enrolled in the study at birth. Calves were kept with their dam for a few hours but systematically received $2 \mathrm{~L}$ of warm colostrum of good quality ( $\geq 50 \mathrm{~g}$ IgG per L) that is conserved at $-20^{\circ} \mathrm{C}$ until use. Calves were individually housed for the first week of life and bottle fed $3 \mathrm{~L}$ of milk twice daily $(0700 \mathrm{~h}$ and $1800 \mathrm{~h})$. After the first week, calves were group housed according to treatment with ad libitum access to water and hay. Calves were fed up to $8 \mathrm{~L}$ of milk per day through the use of an automated milking system (De Laval, Sursee, Switzerland). Similarly, calves had access to calf starter (STARTIVO, Centraliment, Aurillac, France) from four weeks of age with a maximum daily allowance of $2 \mathrm{~kg}$ in the pre-weaning period. In the immediate post-weaning period, calves had access to $2 \mathrm{~kg}$ of GENIE ELEVAGE (Centraliment, Aurillac, France). Chemical composition of dietary ingredients is presented in Table S1.

Calves were randomly assigned at birth to either a treatment (3-NOP, $3 \mathrm{mg} 3-\mathrm{NOP} / \mathrm{kg} \mathrm{BW,} \mathrm{n}=10$ up to week 23, a heifer was removed from the herd due to infertility as a result of being born a twin, i.e., a free-martin, $n=9$ at week 60) or control (CONT, placebo premix containing $\mathrm{SiO}_{2}$ and propylene glycol only, $\mathrm{n}=8$, nine calves were recruited but one calf died early in the study) group, such that breed distribution and birthweight were balanced across groups. The 3-NOP supplement contained 10\% 3-NOP diluted in propylene glycol and adsorbed on $\mathrm{SiO}_{2}$, such that $30 \mathrm{mg}$ of the supplement was fed per kilogram of body weight to achieve the above target dose of 3-NOP. The 3-NOP and placebo were mixed with water $(300 \mathrm{mg} / \mathrm{mL}$ water $)$ and administered daily via an oral gavage approximately $2 \mathrm{~h}$ after feeding. Calves were treated daily from the day of birth, following consumption of colostrum, until 14 weeks of age. All calves were weaned at week 11 using the step-down method over two weeks. After weaning, all calves were group housed in a single pen to replicate normal production practices.

Calves were weighed weekly. Daily individual milk and concentrate intakes prior to weaning were recorded using automated feeders. Total group intake of hay and concentrate, and all refusals in the post-weaning period were recorded twice weekly.

Sampling. All calves were sampled for rumen fluid and faecal content at 1, 4, 11, 14, 23 and 60 weeks of life. Sampling at week 11 was conducted immediately prior to weaning and sampling at week 14 was conducted just prior to cessation of the treatment. Samples of rumen liquid were obtained via oesophageal tubing at least $2 \mathrm{~h}$ after feeding. Aliquots $(1 \mathrm{~mL})$ of rumen liquid were immediately frozen in liquid nitrogen and stored at $-80^{\circ} \mathrm{C}$ until DNA extraction. Additional rumen liquid aliquots were taken for analysis of volatile fatty acids and ammonia as previously described ${ }^{25,26}$. At week $60,3 \mathrm{~mL}$ of ruminal fluid was added to $3 \mathrm{~mL}$ of methyl green formalin saline (MFS) solution ( $35 \mathrm{~mL} / \mathrm{L}$ formaldehyde, $0.14 \mathrm{mM} \mathrm{NaCl}$, and $0.92 \mathrm{mM}$ methyl green) and stored in the dark at room temperature until protozoa were counted. At each sampling period, calves were rectally fingerstimulated with sterile-gloved hand to facilitate the collection of a faecal sample, which was immediately frozen in liquid nitrogen and stored at $-80^{\circ} \mathrm{C}$ until DNA extraction. Blood samples were taken via jugular venepuncture into a heparin tube at week 11, 14 and 23 for metabolic analysis. Blood was immediately centrifuged at $1500 \times g$ for $10 \mathrm{~min}$ at $4{ }^{\circ} \mathrm{C}$. Plasma was stored at $-80^{\circ} \mathrm{C}$ until analysis.

Methane measurements. Methane emissions were recorded using the GreenFeed system (C-Lock Inc., Rapid City, South Dakota, USA) during two time periods. Firstly, from weaning (week 11) until week 23, one GreenFeed system was programmed using C-Lock Inc. software to deliver a maximum of six rotations of a feed dispensing cup, delivering $\sim 6 \mathrm{~g}$ of pellet concentrate GENIE ELEVAGE (as fed) per rotation, with intervals of $30 \mathrm{~s}$ between each rotation, so that $36 \mathrm{~g}$ of pellet was delivered during each visit. During the second phase of measurement when heifers were 57 to 60 weeks of age, two GreenFeed systems were utilised with software 
programmed to deliver a maximum of six rotations of a feed dispensing cup, delivering approximately $45 \mathrm{~g}$ of pellet (as fed) per rotation, with intervals of $30 \mathrm{~s}$ between each rotation, so that $270 \mathrm{~g}$ of pellet was delivered during each visit. During the second measurement period, calves were separated according to treatment group and allocated to one of two GreenFeed systems. An adaptation period of one week preceded a 4-week experimental recording period. After two weeks, calves were rotated into the alternate pen to eliminate any possible biases between the two GreenFeed systems. During all measurement periods, a minimum of $3 \mathrm{~h}$ was required between visits. The calf starter pellets described above were used as an enticement. Recorded methane measurements were included if the total time spent in the feeder was $>3 \mathrm{~min}$ with calves visiting the feeder a minimum of three times per day to ensure repeatability of the recorded measurements ${ }^{27}$.

DNA extraction and amplicon sequencing. Genomic DNA (gDNA) was extracted from each rumen and faeces sample using a bead-beating and on-column purification ${ }^{28}$. DNA extracts were quantified on a Nanodrop 1000 Spectrophotometer (Thermo Fisher Scientific, France) and run on a FlashGel System (Lonza, Rockland, Inc.) to check integrity. Approximately $15 \mu \mathrm{g}$ of rumen or faecal gDNA were sent to Roy J. Carver Biotechnology Center (Urbana, IL61801, USA) for microfluidics PCR amplification (Biomark HD, Fluidigm, South San Francisco, USA) and HiSeq Illumina paired end sequencing. Selected primers for amplification targeting the V3-V5 region of 16S rRNA gene of bacteria, 16S rRNA gene of archaea, fungal ITS2 and protozoal 18S rRNA gene are presented in Table S2. After amplification all samples were pooled. The library was sequenced on one lane of a HiSeq V2 Rapid flowcell for 251 cycles from each end of the fragments using a HiSeq 500-cycle SBS sequencing kit version 2 (Illumina, San Diego, USA).

Bioinformatic analysis. All pipelines have a quality control step, removing sequences with Phred scores of $<25$ and trimming based on expected amplicon lengths, merging paired reads, chimera identification and removal, and OTU picking. Rare OTUs (tripletons or less) were removed from the total OTU dataset. All sequences were submitted to the Sequence Read Archive under BioProject ID PRJNA641997.

Archaeal output fastq files were analysed by downstream computational pipelines (QIIME software v.1.33.2 ${ }^{29}$; length $\geq 250$, maximum five primer mismatches, $<8$ homopolymers). Chimeric sequences were detected using the UCHIME algorithm (USEARCH 6.1) to run both de novo and reference based chimera detection. Sequences were clustered at the $99 \%$ sequence similarity level using GreenGenes v13.8 database using an open referencebased OTU picking approach with the QIIME algorithm and usearch61 method with default parameters ${ }^{30,31}$. For rumen, 2,554,377 reads were obtained, average 20,272 per sample, $87.2 \%$ passed quality control and $7.8 \%$ chimeric sequences were eliminated. The filtered OTU table had 7987 picked OTUs. For faeces, 1,497,269 reads were obtained, average 13,368 per sample, $97.7 \%$ passed quality control and $5.0 \%$ chimeric sequences were eliminated. The filtered table had 1380 picked OTUs.

For bacteria and protozoa and for fungi we followed default settings of IM Tornado and PIPITs pipelines, respectively. Raw bacterial (1,929,957 total and 15,317 average per sample; and 2,760,706 reads, average 24,649 per sample, for rumen and faeces, respectively) and protozoal (1,567,407 total and 12,440 average per rumen sample) reads were analysed using the IM-Tornado pipeline ${ }^{32}$, a tool designed to analyse sequencing data producing two separate reads that do not overlap. For rumen contents $90.2 \%$ and $87.3 \%$ of the reads passed the quality trimming for bacteria and protozoa, respectively; for faecal contents, the good quality reads represented $88.9 \%$ and $91.6 \%$. As reads are not overlapping, IM Tornado is performing analysis simultaneously on Read1, Read 2 and merged R1R2. At the dereplication step, all quality trimmed reads were collapsed into a set of unique reads which accounted for (average of Read1, Read2 and R1R2) $28 \pm 8 \%$ and $25 \pm 8 \%$ in the bacterial rumen and faecal datasets and $30 \pm 9 \%$ and $16 \pm 7 \%$ for the protozoa. In all datasets, chimeric sequences represented less than $1 \%$. Silva database release 123_1 was used in both cases to assign taxonomy. OTU picking resulted in 3551 OTUs for rumen bacteria, 2303 for faecal bacteria, 2303 for rumen protozoa and 465 for faecal protozoa. Faecal protozoa OTUs were not associated to known symbiotic ciliates associated to feed degradation. As a preliminary analysis revealed no differences between groups, they are not further presented in the Results section.

Fungi ITS2 fastq files (4,402,028 total reads and an average 34,937 per sample; and 2,211,525 reads, average 19,746 per sample, for rumen and faeces, respectively) were analysed downstream using the automated PIPITS pipeline (https://github.com/hsgweon/pipits ${ }^{33}$ ). Quality filtered reads represented $96.1 \%$ and $95.4 \%$ in rumen and faecal contents, respectively. In both datasets $82 \%$ of the reads were taxonomically annotated. OTUs were picked using open-reference OTU picking method and the its_12_11_otus reference taxonomy, provided by the UNITE database (https://unite.ut.ee). For rumen 30,100 OTUs were picked and 16,390 OTUs were picked for faeces.

Absolute quantification of archaeal and bacterial gene copies in ruminal contents. Gene copies of 16S rDNA for archaea and bacteria in week 11,14, 23 and 60 weeks of life were quantified using a qPCR approach. Primers used are summarized in Table S2; reaction assay and temperature cycles for archaeal and bacterial $16 \mathrm{~S}$ rDNA were conducted as previously described ${ }^{26,34}$. Triplicate qPCR quantification was performed on $20 \mathrm{ng}$ of extracted gDNA. Amplifications were carried out using SYBR Premix Ex Taq (TaKaRa Bio Inc., Otsu, Japan) on a StepOne system (Applied Biosystems, Courtabeuf, France). Absolute quantification of 16S rDNA copies involved the use of standard curves prepared with gDNA of Methanobrevibacter ruminantium DSM 1093 and Prevotella bryantii DSM 11,371 as described ${ }^{34}$. Results are expressed as $\log _{10}$ gene copies $/ \mathrm{mL}$ of rumen liquid. PCR efficiencies were $104 \%$ and $95 \%$ for archaeal and bacterial $16 \mathrm{~S}$ rDNA, respectively.

Plasma metabolite analysis. Plasma samples ( $100 \mu \mathrm{l})$ for week 14 and 23 were thawed at room temperature and deproteinised with $300 \mu \mathrm{l}$ of cold methanol. After centrifugation $\left(14,000 \times \mathrm{g}, 10 \mathrm{~min},+4{ }^{\circ} \mathrm{C}\right)$, supernatants were evaporated using a Genevac EZ-2 evaporator (Genevac SP Scientific, Ipswich, UK), and dried residues 
were dissolved in 50/50 (v/v) acetonitrile/water containing $0.1 \%$ formic acid. The mixture was transferred to an autosampler vial, and $10 \mu \mathrm{l}$ were injected into a LC-MS/ToF system. To evaluate the system's reproducibility and stability, nine quality-control (QC) samples were prepared by mixing equal volumes of all plasma samples. These QCs were analysed every ten samples, i.e. three times throughout the LC-MS analytical run. Metabolic profiles were then analysed using fast liquid chromatography (1200 Series, Agilent, France) coupled to a timeof-flight mass spectrometer (microTOF, Bruker, Germany). Separation was performed on an Acquity HSS T3 column (Waters, France) using a water/acetonitrile (both containing $0.1 \%$ of formic acid) gradient at a flow rate of $0.4 \mathrm{~mL} / \mathrm{min}$. The linear gradient of $0 \%$ to $100 \%$ of acetonitrile was applied for $13 \mathrm{~min}$ before returning to the initial conditions and equilibrating for $7 \mathrm{~min}$.

The MS system was operated in positive ionization mode with a scan range of $50-800 \mathrm{~m} / \mathrm{z}$. The capillary was set to $-4.5 \mathrm{kV}$, the nebulizer was operated at 2 bars; the dry gas was set to $8 \mathrm{~L} / \mathrm{min}$ at a temperature of $200{ }^{\circ} \mathrm{C}$. The capillary exit was set to $90 \mathrm{~V}$ with skimmer 1 set to $30 \mathrm{~V}$. The time of flight (ToF) was calibrated by using lithium formate (ions at $\mathrm{m} / \mathrm{z} 90$ and 800 ). For accurate mass acquisition, a formate acetate solution was infused during the run at a flow rate of $100 \mu \mathrm{L} / \mathrm{min}$ monitoring for positive ion mode.

Dietary chemical composition. Dietary samples of hay and starter concentrate were ground to pass through a $1-\mathrm{mm}$ sieve and stored at $4{ }^{\circ} \mathrm{C}$ for analysis according to the Association of Official Analytical Chemists ${ }^{35}$. Organic matter was determined by ashing at $550^{\circ} \mathrm{C}$ for $6 \mathrm{~h}$ (Association of Official Analytical Chemists [AOAC] method number 923.03). Crude protein was determined by the Dumas method (CP; N 6.25 ; AOAC method number 992.15). Cell wall components (NDF and ADF) were determined with residual ash (AOAC methods number 200.04 and 973.18) and starch was determined by a polarimetric method ${ }^{36}$.

Statistical analysis. Calf growth, intake, physiological parameters (VFA and methane production) were tested using PROC mixed in SAS 9.4 (SAS Institute Inc., Cary, NC, United States) with repeated measures analysis. Means were compared using the LSMEANS/DIFF statement with treatment, week, and the interaction of treatment $\times$ week as fixed terms; calves nested within treatment as a random effect and week as a repeated measure. The inclusion of breed in the model was not significant and thus, it was not included. Initial and final BW were analysed using a model similar to that described above, but excluding week as a repeated measure. Alpha diversity metrics: Shannon and Simpson diversity indices, richness, and evenness for all microbial communities and qPCR abundance were analysed independently for each time point using the PROC GLM procedure in SAS.

OTUs tables per week and microbial group were uploaded separately in R, and OTU tables were TSS (Total Sum Scaling) normalized after addition of pseudocounts to the whole datasets to deal with zero-values. Subsequent analysis on these normalized tables was performed using mixOmics ${ }^{37}$ on centred log-ratio (CLR) transformed compositional data. Initial PLS-DA analysis was done on 10 components for performance assessment. Distance and number of components were manually tuned by microbial group and by week, after examining the error rates (perf function). Tuning of the sparse-PLS-DA allowed to select the number of components and variables for the final model. Further, in an attempt to explain fermentation parameters and methane production with respect to OTU abundance, heatmaps were made (cim function on mixOmics) based on the sparse-PLS results on three components. The canonical mode was used with a threshold value of 0.7 and default Euclidian distance and Ward linkage.

Microbial community composition was compared across treatments in each week using a t-test and where appropriate a post hoc FDR test was used to eliminate false significant data ${ }^{38}$. For plasma metabolite analysis, MS data were converted to NetCDF format using DataAnalysis 3.4 software (Bruker Biospin, Germany), and processed using a Galaxy web instance workflow for metabolomics (W4M) ${ }^{39}$. The data were first filtered on retention time, and signals outside the range $(<0.4 \mathrm{~min}$ or $>22 \mathrm{~min})$ were removed. Background noise was also removed by subtracting masses found in blank samples (solvents). Signal intensities were then normalized within-batch using a linear regression model ${ }^{40}$. After filtration and normalisation, the number of features was reduced from 2426 to 1175 . A data matrix containing mass and retention time with associated signal intensities for all detected peaks was generated and analysed by multivariate analysis using SIMCA-P software (Umetrics, v. 13.01, Sweden). Principal component analysis (PCA) was performed to look for clusters between treatments or identify potential outliers. A tight clustering of QC samples was obtained in a PCA model indicating stable analytical conditions throughout all measurements.

\section{Results}

Animal growth and rumen physiological parameters remain unchanged. Calf performance data are presented in Table 1. Supplementation with 3-NOP did not affect body weight (BW, kg), average daily gain (ADG), or pre-weaning concentrate or milk intake $(P \geq 0.154)$. Similarly, the inhibitor 3-NOP did not affect individual proportions of rumen volatile fatty acids (VFA; Table S3), or total VFA concentration (mmol/L). Individual proportions of ruminal acetate, propionate, butyrate and the ratio of acetate-to-propionate, in addition to ruminal $\mathrm{NH}_{3}(\mathrm{mM})$ were affected by increasing animal age $(P \leq 0.013$; Table $\mathrm{S} 3)$.

Short-term treatment in early life reduces methane emission from 1-year old calves. Calves receiving 3-NOP showed a persistent reduction in methane emissions $\left(\mathrm{g} \mathrm{CH}_{4} / \mathrm{d}\right)$ throughout the immediate postweaning period and at one year post-weaning, despite treatment ceasing at three weeks after weaning (14 weeks of life; Fig. 1). While calves received 3-NOP in the immediate post-weaning period, methane emissions were $10.4 \%$ lower $(P<0.001)$ in 3 -NOP, compared to CONT calves. A reduction $(P<0.001)$ of $11.9 \%$ persisted until week 23. Subsequently, average methane emissions in the immediate post-weaning period were $11.6 \%$ lower $(P<0.001)$ in 3 -NOP calves, compared to CONT calves $\left(71.2 \pm 1.5\right.$ vs. $80.5 \pm 2.0 \mathrm{~g} \mathrm{CH}_{4} / \mathrm{d}$, respectively). Methane 


\begin{tabular}{|c|c|c|c|c|c|}
\hline \multirow[b]{2}{*}{ Parameter } & \multirow[b]{2}{*}{ CONT } & \multirow[b]{2}{*}{ 3-NOP } & \multicolumn{3}{|c|}{ P-value } \\
\hline & & & TRT & Week & TRT $^{\star}$ Week \\
\hline \multicolumn{6}{|l|}{ Live weight, $\mathrm{kg}$} \\
\hline Birth & $40.8 \pm 3.96$ & $43.4 \pm 3.74$ & 0.154 & & \\
\hline Week 23 & $175.7 \pm 4.34$ & $172.1 \pm 4.17$ & 0.633 & & \\
\hline Week 57 & $361 \pm 12.5$ & $347 \pm 11.8$ & 0.434 & & \\
\hline Week 60 & $382 \pm 11.9$ & $360 \pm 11.2$ & 0.179 & & \\
\hline Week 0 to 23 & $105.7 \pm 3.55$ & $104.4 \pm 3.35$ & 0.807 & 0.001 & 0.997 \\
\hline Week 57 to 60 & $370.1 \pm 9.22$ & $354.2 \pm 8.90$ & 0.235 & 0.320 & 0.422 \\
\hline ADG week 0 to $23, \mathrm{~kg}^{\mathrm{a}}$ & $0.83 \pm 0.04$ & $0.79 \pm 0.04$ & 0.398 & 0.001 & 0.998 \\
\hline ADG week 57 to $60, \mathrm{~kg}$ & $0.76 \pm 0.22$ & $0.56 \pm 0.19$ & 0.476 & 0.034 & 0.689 \\
\hline \multicolumn{6}{|l|}{ Pre-weaning } \\
\hline Concentrate intake, $\mathrm{g} / \mathrm{d}^{\mathrm{b}}$ & $466 \pm 47.3$ & $374 \pm 42.3$ & 0.167 & $<0.001$ & 0.579 \\
\hline Milk intake, $\mathrm{L} / \mathrm{d}$ & $6.57 \pm 0.09$ & $6.45 \pm 0.08$ & 0.212 & $<0.001$ & 0.700 \\
\hline
\end{tabular}

Table 1. Live weight, growth and pre-weaning intake of dairy calves supplemented with $3-\mathrm{NOP}$ ( $3 \mathrm{mg} / \mathrm{kg}$ BW) until 60 weeks of age. Values are presented as means \pm SE. $n=8$ in CONT; $n=10$ in 3-NOP group up to week 23, n =9 for week 57 to 60 . average daily gain, ADG. ${ }^{b}$ Intake of hay was not determined on an individual basis as it was offered to the entire group, concentrate intakes may reflect variability in hay intake in young ruminants.

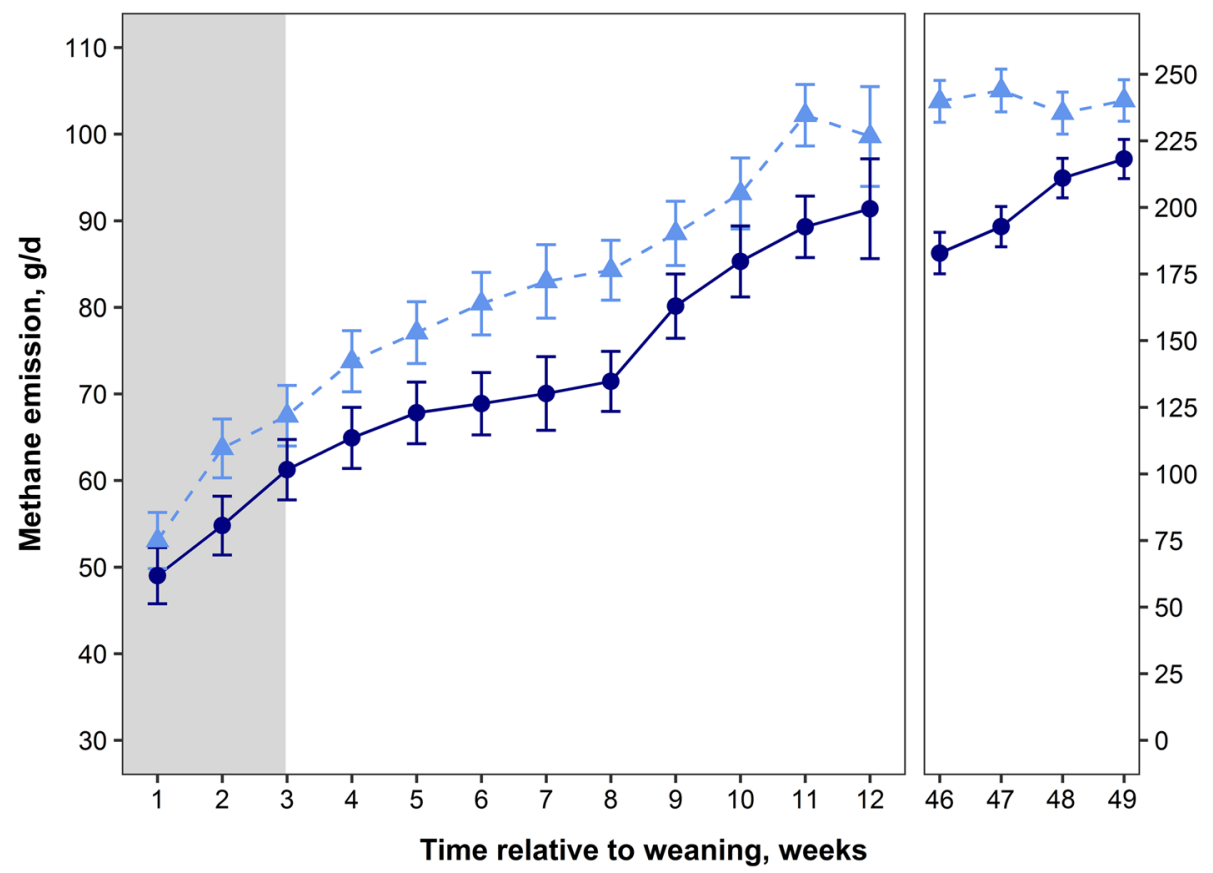

Figure 1. Methane emissions $\left(\mathrm{g} \mathrm{CH}_{4} / \mathrm{d}\right)$ of weaned calves were persistently reduced with supplementation of 3-NOP. Control calves are represented by the light blue dashed line with triangles. Calves supplemented with 3-NOP until 14 weeks of life are represented by the navy blue line with circles. The grey shaded area represents the post-weaning treatment period. Methane emissions were measured using the GreenFeed System (C-Lock, Inc.). Data are treatment means and bars represent SE ( $n=8$ in CONT; $n=10$ in 3-NOP group up to week 23, $\mathrm{n}=9$ for the measurement at 46-49 weeks post-weaning (57-60 weeks of life) due to the removal of a 3-NOP heifer from the herd due to infertility as a result of being born a twin, i.e., a free-martin).

emissions measured around 1 year post-weaning ( 57 to 60 weeks of age) were $17.5 \%$ lower $(P=0.002)$ in heifers treated with $3-\mathrm{NOP}$ as calves, averaging $200.1 \pm 4.2$ versus $242.7 \pm 9.6 \mathrm{~g} \mathrm{CH}_{4} / \mathrm{d}$ in $3-\mathrm{NOP}$ and CONT heifers, respectively. Methane yield $\left(\mathrm{g} \mathrm{CH}_{4} / \mathrm{kg} \mathrm{BW}\right)$ tended $(P=0.08)$ to be $9.2 \%$ lower in 3 -NOP calves during the immediate post-weaning, and was $8.7 \%$ lower at 1 year post-weaning, compared to CONT calves $(P=0.002$; Fig. S1). 
A

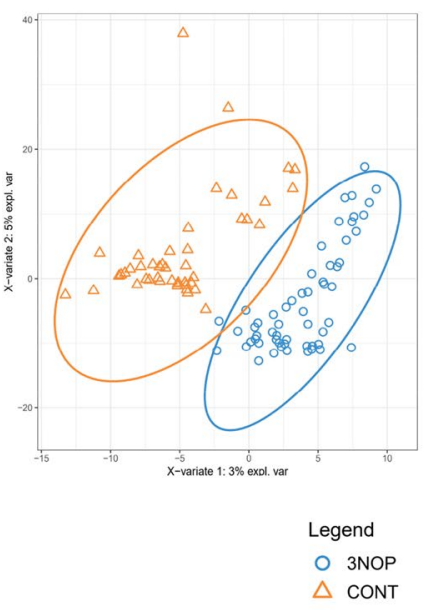

Week 1

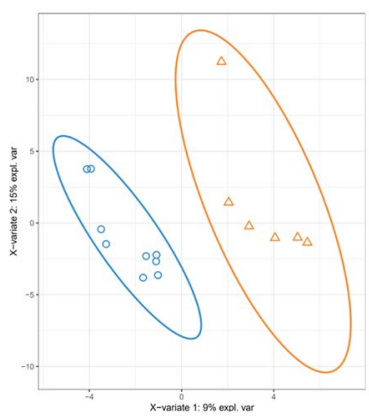

Week 14

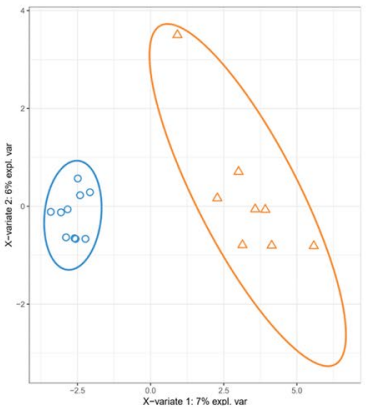

Week 4

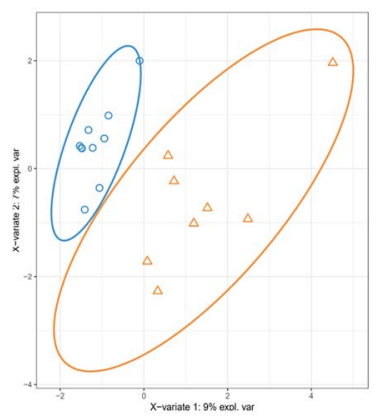

Week 23

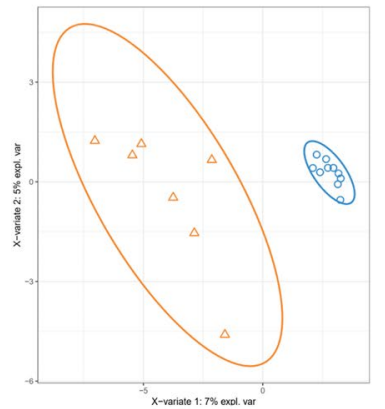

Week 11

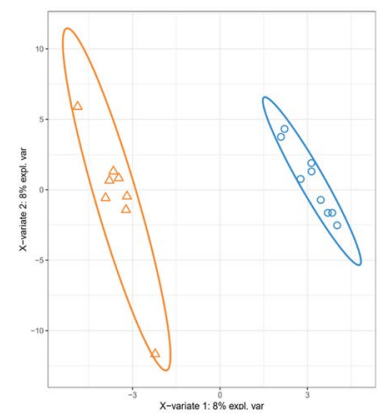

Week 60

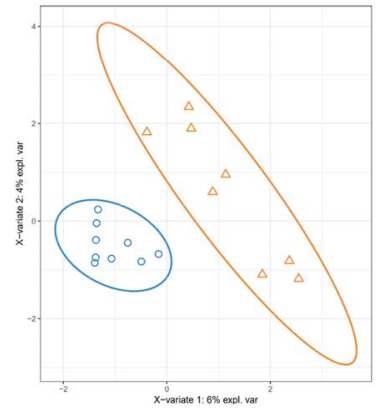

Figure 2. Supplementation of 3-NOP in dairy calves from birth to week 14 of life influences the rumen bacterial community profile. Sparse-PLS-DA loading weights plot of all weeks of ruminal bacteria across treatment groups (CONT, orange vs. 3-NOP, blue) (A). Sparse-PLS-DA revealed separation at all sampling weeks between treatment groups $(\mathrm{CONT}=$ orange and $3-\mathrm{NOP}$ calves $=$ blue $)(\mathbf{B})$.

GIT microbial communities differ according to methane-emission phenotypes. Rumen bacteria and archaea. Bacterial evenness and richness were similar in calves up to 14 weeks of age (Table S4). However, at week 14, the rumen bacterial community in calves supplemented with 3 -NOP was less even $(P=0.031)$ and tended $(P=0.085)$ to have lower richness, compared to CONT calves. Similarly, rumen bacteria in 3-NOP calves had lower richness $(P=0.021)$ at week 60 , despite no differences observed in 23 -week-old calves. Archaeal diversity indices were higher in week 4 for the CONT. Richness was numerically higher in week 11 and 14 with 3-NOP supplementation, but following cessation of treatment, richness was greater in CONT calves at week 23 $(P=0.03)$ and tended $(P=0.08)$ to be greater in week 60. Conversely, archaeal diversity showed lower $(P=0.038)$ evenness in 3-NOP calves at 14 weeks of age, with a similar tendency $(P \leq 0.083$; Table S4) observed in week 4 and 60. Ruminal anaerobic fungi were similar in richness and evenness across all weeks, with the exception of week 11, in which 3-NOP calves tended $(P=0.68)$ to show greater richness of anaerobic fungi, compared to CONT calves (Table S4).

The rumen microbial community structure, visualized using unsupervised PCA, showed separation among bacteria or archaea by week, reflecting the evolution of the community structure with age, but not by treatment group (Fig. S2 A shows plots for bacteria as an example). To further examine the shifting community structure, we used sparse-PLS-DA to identify discriminant OTUs. Though there was some overlap of treatment groups for the bacterial community, the majority of the CONT samples were separated from the 3-NOP samples (Fig. 2A). The separation was evident when considering the effect of treatment within week, as a clear distinction in bacterial community structure was observed between 3-NOP and CONT calves in every week (Fig. 2B and Fig. S2 B for loading plots and heatmaps). The 3-NOP calves showed less dispersion than CONT calves, particularly in weeks 14, 23 and 60 calves treated with 3-NOP formed a tight cluster.

Separation between CONT and 3-NOP calves revealed several discriminant OTUs and most of them were characteristic of CONT, particularly as calves aged (Fig. 2S B). With some exceptions, discriminant OTUs had low relative abundance and varied between weeks due to age and natural changes in diet. As a consequence, there were no common OTUs defining CONT and 3-NOP groups. Correlation to methane emissions were observed in weeks 23 and 60, but not in week 14 when calves were still treated. In week 23, four OTUs classified as Gastranaerophilales representing $2.1 \%$ of sequences were negatively correlated to methane emissions as well as Rikenellaceae_RC9 OTUs that represented $0.7 \%$ of bacterial sequences in week 23 .

Similar sparse-PLS-DA analysis was performed for the rumen archaeal community. However, the fit of the model was unsatisfactory in week 1 , and as such, we omitted these results. The rumen archaeal community 
showed a clear distinction in community structure between 3-NOP and CONT calves when considering calves at all weeks of age, which was further confirmed on a weekly basis (Fig. 3A,B and Fig. S3).

In week 4, OTUs affiliated to Methanobrevibacter gottschalkii clade were discriminant in CONT calves, but OTUs were present at a low abundance. The relative abundance of $M$. gottschalkii clade tended $(P=0.083)$ to be lower in 3-NOP calves, at week 4 (Fig. 3C; Table S6). Yet, M. ruminantium clade tended to be more abundant $(P=0.077)$ in 3-NOP calves aged 4 weeks. CONT calves were characterized by Methanomassiliicoccaceae Group 9 representing $7.3 \%$ of archaeal sequences compared to $0.8 \%$ in 3 -NOP calves $(P=0.002)$. In weeks 11,14 and 23 the model identified several discriminant OTUs in CONT and 3-NOP calves, most were associated with the M. gottschalkii clade and were linked to both groups of calves. Other discriminant OTUs were associated to $M$. ruminantium clade and Methanomassiliicoccaceae Groups 8, 9, 10 and 12 and these were preferentially linked to CONT calves. In contrast, in week 60 only a single unknown Methanobrevibacter OTU was discriminant of CONT calves. As for bacteria, correlations with methane emissions were observed in weeks 23 and 60 . It is noted that in week 60 three Methanomicrobium mobile representing 3\% of archaeal sequences correlated to low methane emissions. Also OTUs associated to Methanomassiliicoccaceae Group 8 were correlated to low methane emissions in week 60 (Fig. S4). M. mobile was only detected in week 60 and was more prevalent in 3-NOP calves $(P<0.05$, Table S6).

Quantification of total ruminal archaea and bacteria by qPCR revealed no statistical differences between treatment groups, in any week (Fig. S5). Similarly, the ratio of archaea-to-bacteria was similar with respect to treatment, across all weeks. However, at 60 weeks of age calves supplemented with 3 -NOP had a numerically lower number of Archaea (4.90 vs. $5.08 \pm 0.04 \log _{10}$ copies $/ \mathrm{mL}$ ) and ratio of archaea-to-bacteria, compared to CONT calves, respectively ( 0.75 vs. $0.8 \pm 0.01 \log _{10}$ copies/mL; Fig. S5).

Rumen fungi and protozoa. Rumen anaerobic fungi showed separation between CONT and 3-NOP calves, particularly in weeks 11, 23 and 60 using sparse-PLS-DA (Fig. S6 A). In week 4, four OTUs affiliated to the Neocallimastigaceae family were discriminant of CONT calves but with low abundances. Conversely, several Caecomyces communis OTUs, some with high abundances were associated with 3-NOP calves. In week 11, discriminant OTUs were more diversified in term of taxa and were common across both treatments. After weaning, in week 14, 23, and 60 there were a reduced number of discriminant OTUs (eight or less) that were predominantly associated to with CONT calves (Fig. S6 B). When OTUs are compiled at genera level no differences were observed between groups (Table S6 and Fig. S7). Most OTUs were only assigned at higher taxonomic levels and are not further presented. However, regarding methane, the most abundant discriminant OTUs linked to greater emissions were associated to the genus Cyllamyces at 23 weeks and to Orpinomyces at week 60.

Ruminal protozoa were only observed at weeks 23 and 60. No differences in alpha-diversity indicators (Table S4) or on counting at 60 weeks (Table S7) were observed between groups. In week 23 the community was dominated by Entodinium spp. and was more diversified in older calves at week 60 (Fig. S8 A). The relative abundance of protozoal Ophryocolex and unclassified Trichostomatia were greater $(P \leq 0.036)$ in CONT, compared to 3-NOP calves in week 23 (Table S6). In week 60, Diplodinium tended $(P=0.092)$ to be more abundant in 3-NOP calves, compared to the CONT. Notwithstanding, sparse-PLS-DA analysis did not show separation between groups (Fig. S8 B).

Faecal microbiota. Faecal bacteria are only presented from week 4, due to the low number of samples for 1 week old calves resulting in an unsatisfactory statistical model. Faecal bacteria showed similar $(P>0.05)$ richness and evenness until week 60 , where 3 -NOP tended $(P=0.068)$ to have greater evenness (Table S5). Sparse-PLS-DA showed bacteria from CONT and 3-NOP calves overlapped in week 4, but from week 11 to week 23 showed clear separation between CONT and 3-NOP calves (Fig. S9 A). Bacteria from week 60 would not converge into a satisfactory sparse-PLS-DA model (not shown), instead we report week 60 bacteria using PLS-DA, which also showed clear separation between treatment groups (Fig. S9 B). In week 14, Rikenellaceae RC9, Bacteriodales and Alloprevotella were discriminant of the CONT and present at very high abundance. Conversely, 3-NOP was characterised by Porphyromonadaceae (OTU \#18) representing 3.28\% of sequences. Whereas in week 23, different OTUs belonging to Ruminococcaceae and Rikenellaceae $R C 9$ families were discriminant, but a higher number of sequences for each taxa were present for CONT and 3-NOP, respectively.

The quality of data was not appropriate to perform PLS-DA analysis on faecal archaeal OTUs (data not shown). However, unclassified archaea and unclassified Euryarchaeota comprised the greatest proportion of faecal archaea averaging $38 \%$ across all weeks, but varying $(P \leq 0.019)$ between CONT and 3-NOP calves in weeks 4 and 11; and 11 and 14, respectively (Table S8). The relative abundance of $M$. gottschalkii clade was greater $(P \leq 0.016)$ in CONT calves, compared to 3 -NOP calves in week 4 and 14 , but was similar in all other weeks. Similarly, M. ruminantium was greater $(P \leq 0.026)$ in CONT calves, compared to 3-NOP calves in weeks 14 and 60 .

Faecal anaerobic fungi in weeks 1 and 4 exhibited a limited number of reads and are therefore not reported. Faecal anaerobic fungi showed no effects of treatment on any alpha-diversity measures (Table S5) or relative abundance (Table S8). Similarly, sparse-PLSDA showed no separation with treatment across week 14 to 60 (data not shown).

Co-occurrence patterns of ruminal microbes differ by treatment. The pattern of microbial cooccurrence in the rumen varied in calves fed CONT versus 3-NOP within each week (Fig. 4). Bacterial taxa Fibrobacter showed a strong positive association with Treponema $(P<0.05)$ in $3-$ NOP calves in weeks 11 and 14 , but these co-occurrences disappeared after the cessation of 3-NOP treatment and no association between these two taxa were observed in week 23 or 60 of life, or in CONT calves at any age (Fig. 4). 
A

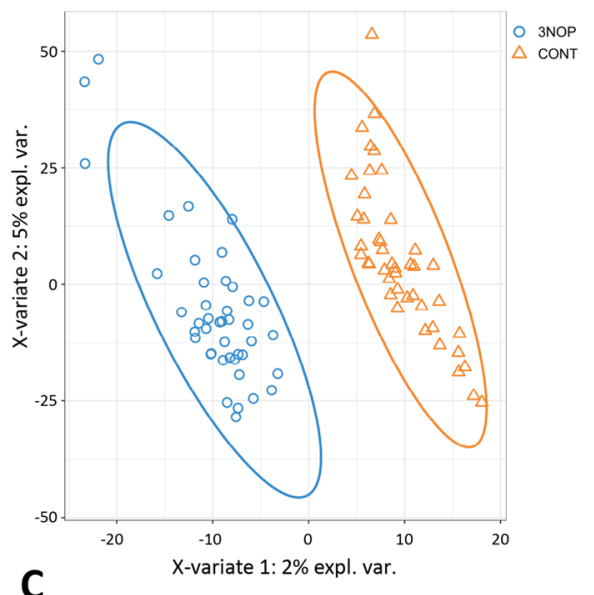

C

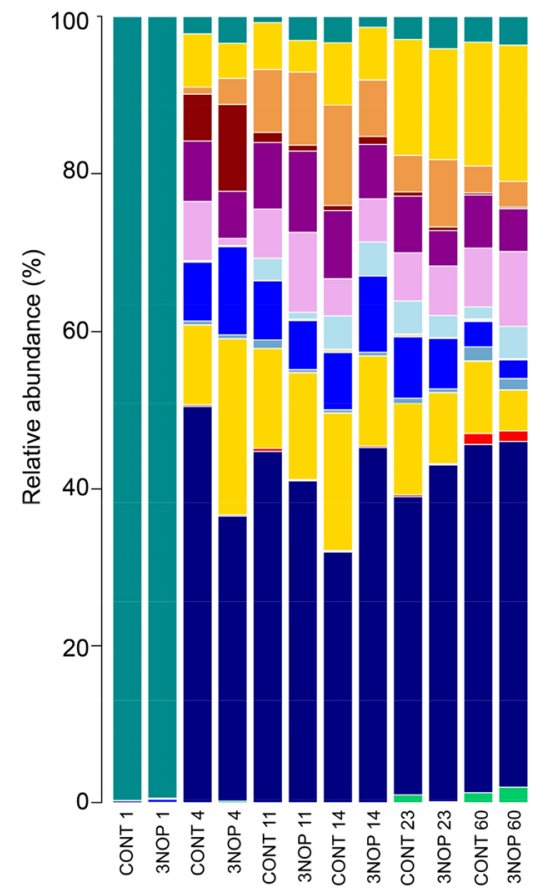

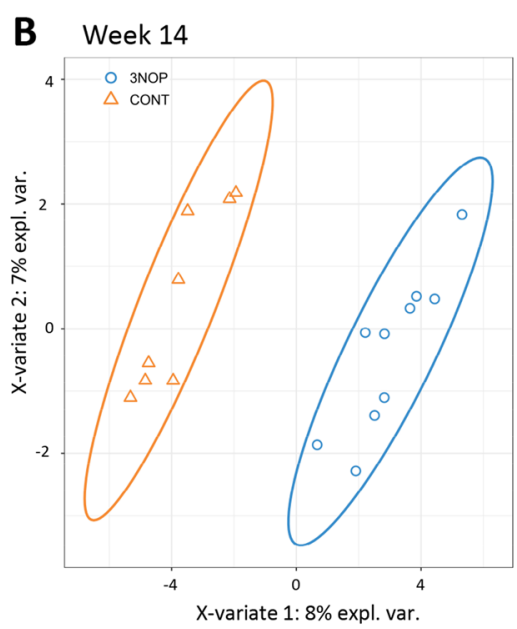

Week 23
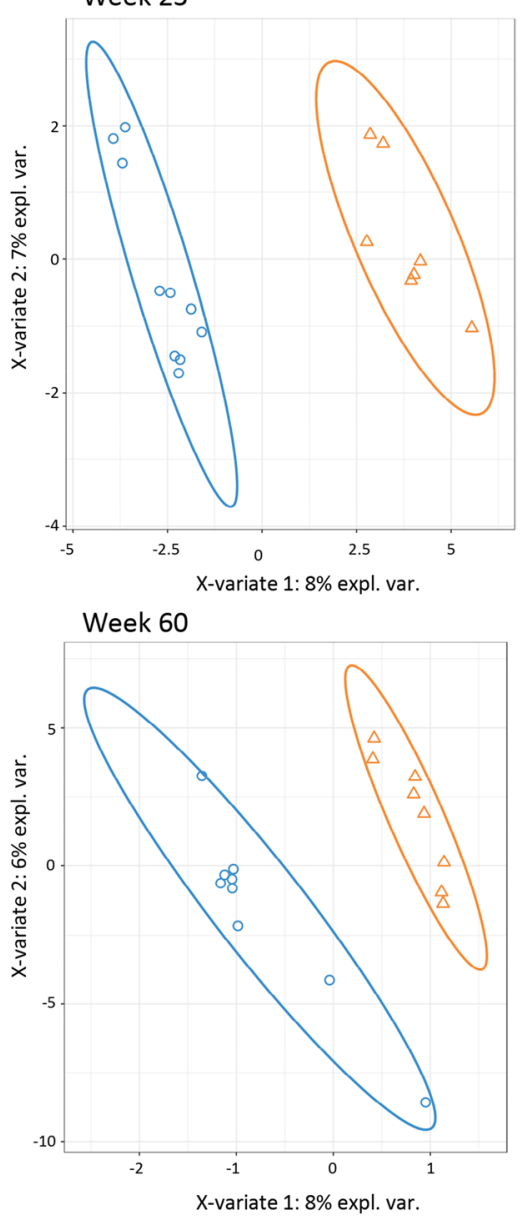

Figure 3. Ruminal archaeal structure differed with 3-NOP supplementation of calves from birth. SparsePLS-DA loading weights plot of archaeal structure across all weeks of age showing separation of the two treatment groups, blue $=3$-NOP, and orange $=$ CONT $(\mathbf{A})$; Sparse-PLS-DA loading weights plots of archaeal microbial structure at week 14, 23 and 60 (B), showing clear separation of treatment groups; Relative abundance of archaeal taxa across all weeks (C).

In the archaeal domain, $M$. ruminantium and $M$. gottschalkii clade exhibited a strong negative association in all weeks $(P \geq 0.05)$ regardless of treatment. With these exceptions, no other common associative patterns were observed across weeks. 

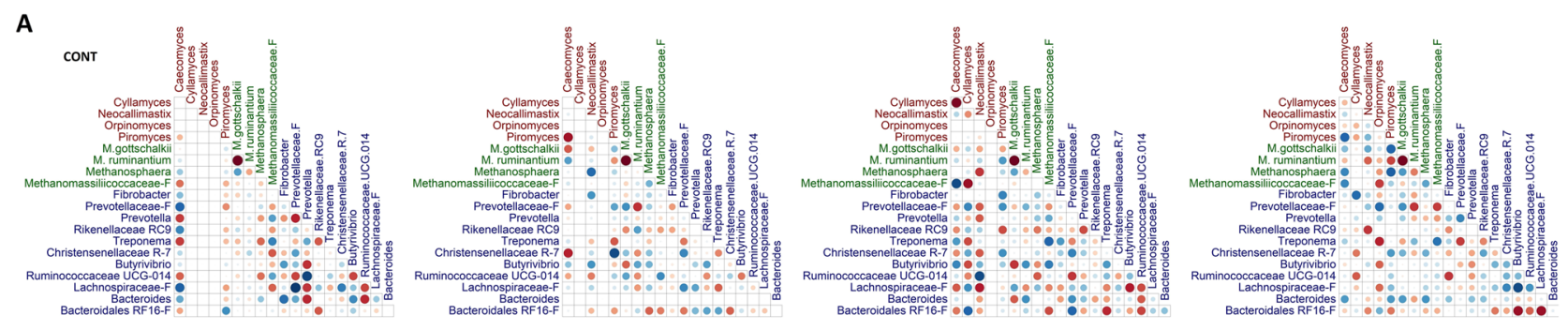

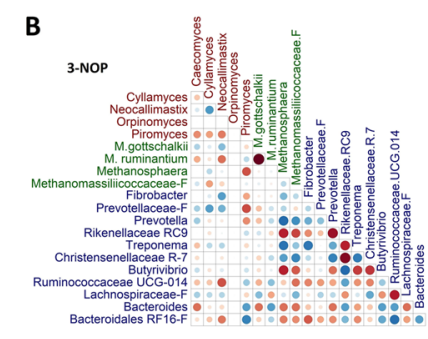

Week 11

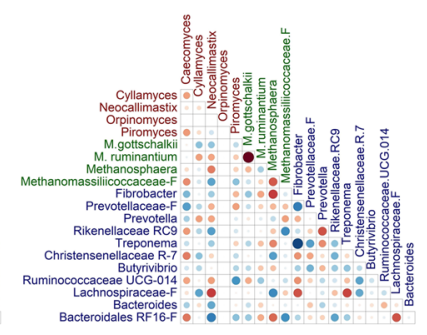

Week 14

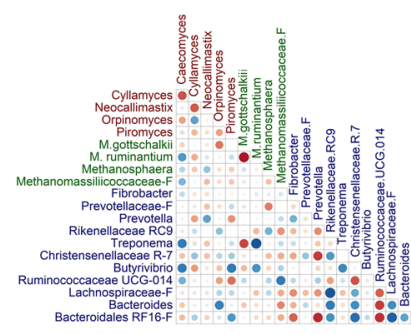

Week 23

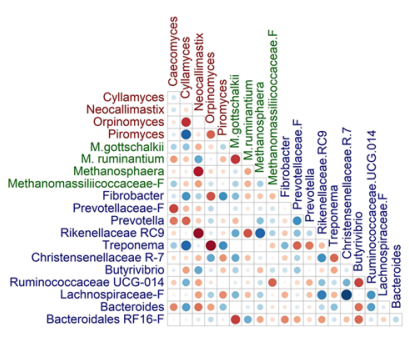

Week 60

Figure 4. Co-occurrence of ruminal genera differed in CONT versus 3-NOP calves at different ages. Circles represent relationships (positive, blue circles; negative, red circles) between taxa abundance in CONT (A) and 3-NOP (B) calves, determined using a Spearman non-parametric rank correlation matrix. Larger circles represent stronger correlations. Anaerobic fungi are displayed in red, archaeal taxa in green, and bacteria in blue.

Comparing between domains revealed differences in the number of significant associations in CONT calves, compared to 3-NOP calves. Calves supplemented with 3-NOP exhibited a mean value of $12.0 \pm 0.58$ out of a possible 190 associations within and between archaeal, bacterial and fungal taxa each week. Conversely, CONT calves exhibited $8.8 \pm 1.11$ associations across these domains each week. Interestingly, 3 -NOP calves had a greater number of significant associations among bacterial taxa each week (mean 8.3 \pm 0.88 ), compared to in CONT (mean $3.3 \pm 0.67$ ) or across domains for week 11,14 and 23. Yet in week 60, CONT calves had 9 significant associations among bacterial taxa, compared to 3-NOP calves which had 3, resulting in a shift towards an increased number of associations between bacterial and fungal taxa in 3-NOP calves.

Plasma metabolite profile. The plasma metabolome of CONT and 3-NOP calves was compared at week 14 and 23 by untargeted LC-MS-TOF. In week 14, a PLS-DA model revealed a separation between CONT and 3-NOP calves (Fig. S10 A). The model was acceptable and explained $41 \%\left(\mathrm{R}^{2} \mathrm{X}\right)$ of variation and had a cross validated predictive ability $\left(\mathrm{Q}^{2}\right)$ of $56 \%$. Conversely, the model in 23 week old calves was poor with no separation between CONT and 3-NOP (Fig. S10 B; $\mathrm{Q}^{2}=0.05$ ). Metabolites with variable importance in projection (VIP) values higher than $1(n=58)$ were considered important in discriminating between control and treated calves at 14 weeks. Nine putative candidates were obtained after querying an in-house database containing more than 1000 metabolites analysed under the same conditions. Identification at level 1 , confirmed by analytical standards, was obtained for methionine, leucine/isoleucine, tyrosine, and phenylalanine. Whereas, tryptophan, cholic acid glycocholic acid, lyso-phosphatidylcholine (lysoPC16:1) and lyso-phosphatidylethanolamine (lysoPE 18:2) were identified at level 2 (Table S9). The concentration found for these nine metabolites at 14 and 23 weeks is shown in Fig. S11.

\section{Discussion}

The persistence of methane inhibition in calves up to at least one year of age, after 3-NOP treatment ceased at 14 weeks, indicates a robust imprinting effect of the early life intervention. Previous studies have illustrated similar potential. Abecia et al. ${ }^{9}$ showed modifications to the microbiome in goats remained 3 months post-treatment, when the doe was treated in addition to the offspring until two months post-weaning. Similarly, De Barbieri et al. ${ }^{41}$ showed that inoculation of lambs with rumen fluid from sheep in the first 2 weeks of life had effects on rumen bacterial communities lasting up to 5 months of age. However, our study is the first to show successful imprinting for reduced methane emissions. Previous studies on adult ruminants showed reductions in methane emissions using 3-NOP for up to 12 weeks in lactating dairy cows ${ }^{18}$, and 16 weeks in beef cattle ${ }^{21}$. However, once treatment ceased, methane emissions of treated animals or in vitro samples ${ }^{42}$ immediately converged toward the control, eliminating the favourable effects. The differences are not surprising due to the highly resilient nature of the mature ruminal microbiome and the strong host-microbiome interaction, which forces the reversion of the microbial population back to its pre-intervention state ${ }^{43}$. As such, an important contributor to the efficacy of the current intervention was targeting the period of microbial establishment, as well as the weaning transition. These comprise periods of dramatic dietary changes and substantial ruminal maturation and colonization, with large shifts in the rumen and faecal microbiomes ${ }^{44,45}$ and functionality. By targeting both transitional periods, 3-NOP supplementation was able to modulate the rumen environment in dairy calves at a crucial period of development 
to elicit long lasting effects on methane output. Similarly, the singularity of this result may come from the choice of supplement, due to its very specific microbial target, the active $\mathrm{Ni}(\mathrm{I})$ site of the methyl-coenzyme $\mathrm{M}$ reductase (MCR) common to all methanogen ${ }^{20}$ that has no redundancy in the ecosystem to compensate its inhibition.

Dietary supplementation of 3-NOP in ruminant livestock has successfully reduced methane emissions in dairy and meat production systems by a mean of 20 to $40 \%{ }^{46}$. Importantly, digestibility and production remain largely unaffected by the supplement. Here, methane emissions of dairy calves immediately post-weaning and at one year post-weaning were successfully reduced, whilst the fermentative capacity of the rumen was maintained with 3-NOP supplementation. Evidenced here, and previously, as $\mathrm{DMI}^{47}$, ammonia concentration ${ }^{48}$, total VFA ${ }^{42}$, and proportions of propionate and acetate were unaltere ${ }^{49}$. This contrasts previous reports of a reduced A:P ratio with supplementation of 3-NOP ${ }^{42,47,48}$. A possible explanation may be that most studies report larger methane reductions than that observed here. Other explanation could be the overriding effect of calf age and development, where the associated variability in forage:milk:concentrate intake of young calves and establishing microbial structure diminished any treatment effect. Further, hay intake in the young calves was not able to be quantified in the current study, potentially explaining the large variation observed in concentrate intake. Similarly, variability in the degree of methane inhibition observed with 3-NOP is influenced by the method of administration, timing relative to feeding, and the basal diet. The magnitude of methane inhibition (mean 13.1\%) in this study from week 11 to 14 during supplementation of weaned calves reinforces that timing of product administration relative to feeding influences the observed effect. The largest reductions have been observed with direct 3-NOP inclusion into the feed ensuring continuous ingestion throughout the meal, in comparison to its application directly into the rumen of cannulated cows ${ }^{48}$.

Yet, as a calf's digestive tract functions as a pseudo-monogastric in early life, the ideal method of administration, being as a mixture with milk, would induce a reflexive closure of the oesophageal groove, shunting the supplement passed the rumen. We administered the treatment via oral gavage $2 \mathrm{~h}$ after milk feeding as a mixture with a minimal amount of water to minimize this instinctive response and to maximise ruminal flow ${ }^{50,51}$. Subsequently, it is possible that the observed effects could be enhanced if an alternative method of administration to young calves is devised. The oesophageal groove reflex is not always stimulated and depending on how milk is provided it can potentially fall in the forestomach ${ }^{51}$. Another approach may be the additional inclusion of 3-NOP with solid feeds; however, this would delay supplementation until calves are $\sim 3-4$ weeks of age with a highly inconsistent rate of consumption in the initial period, potentially diminishing any effect on the establishment of the ruminal microbiome.

Nevertheless, distinct microbial structures were visible in both the rumen and faeces of CONT and 3-NOP calves, even after cessation of 3-NOP supplementation and group mixing of calves. This indicates a persistent change in the GIT microbiota by the early life intervention. The absence of a consistent modification to the GIT microbiome likely results from a stronger effect of increasing age on microbial community development, than the effect of treatment ${ }^{45}$. Yet, there were some microbial indicators that are known to be associated to enteric methane production. A clear positive association was observed only between Fibrobacter and Treponema in 3-NOP calves during the treatment period, suggesting a direct impact of 3-NOP on the microbial interactions within the rumen. This altered association between bacterial taxa linked to minimal $\mathrm{H}_{2}$ production (Fibrobacter), and reduced methane emissions (Treponema; as observed by Cunha et al., ${ }^{52}$ ), provides support for the effects of 3-NOP to alter the rumen microbial community in favour of a low-methane emitting phenotype. The continued reduction in methane emissions from 3-NOP calves, post-treatment, potentially resulted from an additional suppression of bacterial taxa known to be high $\mathrm{H}_{2}$ producers ${ }^{53}$ including numerical reductions on the overall abundance of members of Ruminococcaceae, Christensenellaceae and Lachnospiraceae families. This is in agreement with observations by Ramayo-Caldas et al. ${ }^{54}$ in Holstein cattle and Kittelman et al ${ }^{55}$ in sheep, where higher abundances of Ruminococcaceae, Coprococcus, Lachnospiraceae, and Christensenellaceae were linked to higher methane emissions.

Similarly, a negative association between Fibrobacter and M. gottschalkii clade in week 23 in 3-NOP calves (Fig. 4) supports this, as the production of succinate rather than $\mathrm{H}_{2}$ from degradation of cellulose could be expected to reduce the abundance of the hydrogenotrophic M. gottschalkii clade. Similar findings have been reported previously, where a lower presence of M. gottschalkii clade was linked to lower methane emissions in cattle $^{38}$. Yet, Ramayo-Caldas et al. ${ }^{54}$ noted a lack of clear association between M. gottschalkii clade and high or low methane emitting Holstein dairy cows. Importantly, the reliance on bacteria for $\mathrm{H}_{2}$ can be inverted, where some species would utilize excess $\mathrm{H}_{2}$ available following the supplementation of 3-NOP ${ }^{56}$ reflecting the various pathways through which metabolic hydrogen can be redirected, including formate, succinate, ethanol, microbial biomass and reductive acetogenesis ${ }^{42}$. It was reported that 3-NOP has a greater negative effect on methylotrophic methanogens than on hydrogenotrophic methanogen $s^{57}$. This effect was not observed in our study and contrasts with the higher prevalence of Methanomassiliicoccaceae Group 8 that were correlated to lower methane emissions in 3-NOP calves at 60 weeks of age, after treatment ceased.

Such associations between archaea and bacteria also have implications for the reported relationship between the ratio of total archaea to bacteria and methane output and their variability may help explain inconsistencies in the relationship. Due to their key role in methanogenesis, a reduced archaeal abundance would be expected to lower methane emissions. However, this relationship has proven elusive in some studies ${ }^{55,58-60}$, with others reporting only weak correlations ${ }^{61}$. Data on the effects of 3-NOP on rumen microbial structure are scarce and inconsistent. Supplementation of 3-NOP in 6 ruminally-cannulated late-lactation Holstein cows failed to alter methanogenic archaea (Methanobrevibacter, Methanosphaera, and Methanomicrobium spp.) and bacterial cell counts in whole ruminal contents despite a $31 \%$ reduction in methane, compared to the control ${ }^{49}$, in agreement with the current results for total quantification of archaea and bacteria by qPCR. However, we noted a numerical reduction in archaeal copy number in 3-NOP calves, compared to CONT calves suggesting that despite a lack of significance potentially resulting from large variation between individual animals, 3-NOP was able to reduce 
the archaeal population in the rumen at 60 weeks of life. Romero-Pérez et al. ${ }^{62}$ noted no effect of 3-NOP on the total copy number of $16 \mathrm{~S}$ rRNA genes for methanogens and bacteria in the liquid phase of a RUSITEC, but reported a large decrease in the solid associated methanogens. As the current study was unable to capture solid associated microbes, this may provide an avenue for further investigation. Interestingly, there was an apparent fauna-free status of all calves in the early weeks of life, with protozoa only appearing at week 23 and being present in week 60 of life, likely due to their isolation from adult animals from birth. As protozoa conduct interspecies $\mathrm{H}_{2}$ transfer with methanogens, defaunation could potentially reduce methane emissions by $10.5 \%{ }^{63}$, yet such an effect would be expected across both CONT and 3-NOP calves, in the current study.

As protozoa, rumen fungi are also involved in interspecies $\mathrm{H}_{2}$ transfer with methanogens ${ }^{64}$. They also produce formate, another common substrate for methane synthesis, and might have a role in overall enteric methane emissions ${ }^{65}$. Similarly to bacteria and archaea, the fungal community structure differed between CONT and 3-NOP calves but no divergence in their abundance was detected. Unfortunately, the changes observed in OTUs and correlations to fermentation parameters cannot be associated to the methane phenotype without additional studies.

Metabolomic analysis of plasma revealed a separation between CONT and 3-NOP calves at week 14, while calves were still being treated. However, following cessation of treatment, calves aged 23 weeks showed no separation. This was an exploratory analysis as no additional identification studies were done, but highlights that differences in rumen methane emissions can be reflected in plasma metabolites. It is noted that many of the differences in discriminant metabolites between groups at week 14 showed similar tendencies at week 23 (Fig. S11). Studies with larger number of animals should confirm whether or not differences in metabolic profiles may persist post-treatment.

\section{Conclusion}

Our study examined the neonatal calf up to 60 weeks of life, a period in which the relationship between enteric methane emission and microbial structure has been largely overlooked. We observed a long-term persistent reduction in methane emissions from calves up to 1 year of life following supplementation with 3-NOP from birth, through weaning, to 14 weeks of life. This accounted for an accumulated reduction of circa $150 \mathrm{~kg}$ of $\mathrm{CO}_{2} \mathrm{eq}$ that were not released into the environment by calves in their first year of life. In the same way, the ruminal and faecal microbiota of treated and control calves exhibited a clear differentiation, particularly for bacteria. It is apparent that certain microbial taxa were associated with methane emissions. Notwithstanding, contrasting increases or decreases were observed across weeks, indicating that further study is needed to determine the exact role of each taxa, or perhaps that certain taxa are able to modulate their function under certain circumstances. The presence of similar characteristic taxa driving the separation of treatment groups across faeces and ruminal microbiota, and their presence in previous studies, suggests these taxa may be key determinants of methane emissions from dairy calves.

Received: 22 September 2020; Accepted: 15 January 2021

Published online: 04 February 2021

\section{References}

1. Gerber, P. J. et al. Tackling Climate Change Through Livestock-A Global Assessment of Emissions and Mitigation Opportunities (Food and Agriculture Organization of the United Nations (FAO), Rome, 2013).

2. Johnson, K. A. \& Johnson, D. E. Methane emissions from cattle. J. Anim. Sci. 73, 2483-2492. https://doi.org/10.2527/1995.73824 83x (1995)

3. Weimer, P. J., Stevenson, D. M., Mantovani, H. C. \& Man, S. L. C. Host specificity of the ruminal bacterial community in the dairy cow following near-total exchange of ruminal contents. J. Dairy Sci. 93, 5902-5912 (2010).

4. Li, R. W., Connor, E. E., Li, C., Baldwin Vi, R. L. \& Sparks, M. E. Characterization of the rumen microbiota of pre-ruminant calves using metagenomic tools. Environ. Microbiol. 14, 129-139. https://doi.org/10.1111/j.1462-2920.2011.02543.x (2012).

5. Jami, E., Israel, A., Kotser, A. \& Mizrahi, I. Exploring the bovine rumen bacterial community from birth to adulthood. ISME J. 7, 1069-1079. https://doi.org/10.1038/ismej.2013.2 (2013).

6. Rey, M. et al. Establishment of ruminal bacterial community in dairy calves from birth to weaning is sequential. J. Appl. Microbiol. 116, 245-257. https://doi.org/10.1111/jam.12405 (2014).

7. Fonty, G., Gouet, P., Jouany, J. P. \& Senaud, J. Establishment of the microflora and anaerobic fungi in the rumen of lambs. J. Gen. Microbiol. 133, 1835-1843 (1987).

8. Morvan, B. et al. Establishment of hydrogen-utilizing bacteria in the rumen of the newborn lamb. FEMS Microbiol. Lett. 117, 249-256. https://doi.org/10.1016/0378-1097(94)90567-3 (1994).

9. Abecia, L. et al. An antimethanogenic nutritional intervention in early life of ruminants modifies ruminal colonization by Archaea. Archaea 2014, 841463. https://doi.org/10.1155/2014/841463 (2014).

10. Newbold, C. J., Wallace, R. J. \& McIntosh, F. M. Mode of action of the yeast Saccharomyces cerevisiae as a feed additive for ruminants. Br. J. Nutr. 76, 249-261. https://doi.org/10.1079/bjn19960029 (1996).

11. Desnoyers, M., Giger-Reverdin, S., Bertin, G., Duvaux-Ponter, C. \& Sauvant, D. Meta-analysis of the influence of Saccharomyces cerevisiae supplementation on ruminal parameters and milk production of ruminants. J. Dairy Sci. 92, 1620-1632. https://doi. org/10.3168/jds.2008-1414 (2009).

12. McSweeney, C. S., Palmer, B., McNeill, D. M. \& Krause, D. O. Microbial interactions with tannins: nutritional consequences for ruminants. Anim. Feed Sci. Technol. 91, 83-93. https://doi.org/10.1016/S0377-8401(01)00232-2 (2001).

13. Patra, A. K. Meta-analyses of effects of phytochemicals on digestibility and rumen fermentation characteristics associated with methanogenesis. J. Sci. Food Agric. 90, 2700-2708. https://doi.org/10.1002/jsfa.4143 (2010).

14. Patra, A. K. \& Saxena, J. The effect and mode of action of saponins on the microbial populations and fermentation in the rumen and ruminant production. Nutr. Res. Rev. 22, 204-219. https://doi.org/10.1017/S0954422409990163 (2009).

15. Ramos-Morales, E. et al. Antiprotozoal effect of saponins in the rumen can be enhanced by chemical modifications in their structure. Front. Microbiol. https://doi.org/10.3389/fmicb.2017.00399 (2017). 
16. Knight, T. et al. Chloroform decreases rumen methanogenesis and methanogen populations without altering rumen function in cattle. Anim. Feed Sci. Technol. 166-167, 101-112. https://doi.org/10.1016/j.anifeedsci.2011.04.059 (2011).

17. Abecia, L. et al. Effect of bromochloromethane on methane emission, rumen fermentation pattern, milk yield, and fatty acid profile in lactating dairy goats. J. Dairy Sci. 95, 2027-2036. https://doi.org/10.3168/jds.2011-4831 (2012).

18. Hristov, A. N. et al. An inhibitor persistently decreased enteric methane emission from dairy cows with no negative effect on milk production. Proc. Natl. Acad. Sci. USA https://doi.org/10.1073/pnas.1504124112 (2015).

19. Popova, M. et al. Changes in the rumen microbiota of cows in response to dietary supplementation with nitrate, linseed, and saponin alone or in combination. Appl. Environ. Microbiol. 85, e02657-e12618. https://doi.org/10.1128/aem.02657-18 (2019).

20. Duin, E. C. et al. Mode of action uncovered for the specific reduction of methane emissions from ruminants by the small molecule 3-nitrooxypropanol. Proc. Natl. Acad. Sci. USA 113, 6172-6177. https://doi.org/10.1073/pnas.1600298113 (2016).

21. Romero-Perez, A. et al. Sustained reduction in methane production from long-term addition of 3-nitrooxypropanol to a beef cattle diet. J. Anim. Sci. 93, 1780-1791. https://doi.org/10.2527/jas2014-8726 (2015).

22. Romero-Perez, A. et al. The potential of 3-nitrooxypropanol to lower enteric methane emissions from beef cattle. J. Anim. Sci. 92 , 4682-4693. https://doi.org/10.2527/jas.2014-7573 (2014).

23. Hristov, A. N. et al. The use of an automated system (GreenFeed) to monitor enteric methane and carbon dioxide emissions from ruminant animals. J. Vis. Exp. 103, 52904. https://doi.org/10.3791/52904 (2015).

24. Jayanegara, A. et al. Use of 3-nitrooxypropanol as feed additive for mitigating enteric methane emissions from ruminants: a metaanalysis. Ital. J. Anim. Sci. 17, 650-656. https://doi.org/10.1080/1828051x.2017.1404945 (2017).

25. Rira, M. et al. Potential of tannin-rich plants for modulating ruminal microbes and ruminal fermentation in sheep. J. Anim. Sci. 93, 334-347. https://doi.org/10.2527/jas.2014-7961 (2015).

26. Morgavi, D. P. et al. Rumen microbial communities influence metabolic phenotypes in lambs. Front. Microbiol. 6, 1060. https:// doi.org/10.3389/fmicb.2015.01060 (2015).

27. Arbre, M. et al. Repeatability of enteric methane determinations from cattle using either the SF6 tracer technique or the GreenFeed system. Anim. Prod. Sci. 56, 238-243. https://doi.org/10.1071/an15512 (2016).

28. Popova, M., Martin, C. \& Morgavi, D. Improved protocol for high-quality co-extraction of DNA and RNA from rumen digesta. Folia Microbiol. 55, 368-372. https://doi.org/10.1007/s12223-010-0060-3 (2010).

29. Schloss, P. D. et al. Introducing mothur: open-source, platform-independent, community-supported software for describing and comparing microbial communities. Appl. Environ. Microbiol. 75, 7537-7541. https://doi.org/10.1128/AEM.01541-09 (2009).

30. Caporaso, J. G. et al. PyNAST: a flexible tool for aligning sequences to a template alignment. Bioinformatics 26, 266-267. https:// doi.org/10.1093/bioinformatics/btp636 (2010).

31. Caporaso, J. G. et al. QIIME allows analysis of high-throughput community sequencing data. Nat. Methods 7, 335-336. https:// doi.org/10.1038/nmeth.f.303 (2010).

32. Jeraldo, P. et al. IM-TORNADO: a tool for comparison of $16 \mathrm{~S}$ reads from paired-end libraries. PLoS ONE 9, e114804. https://doi. org/10.1371/journal.pone.0114804 (2014).

33. Gweon, H. S. et al. PIPITS: an automated pipeline for analyses of fungal internal transcribed spacer sequences from the Illumina sequencing platform. Methods Ecol. Evol. 6, 973-980. https://doi.org/10.1111/2041-210x.12399 (2015).

34. Popova, M. et al. Effect of fibre- and starch-rich finishing diets on methanogenic Archaea diversity and activity in the rumen of feedlot bulls. Anim. Feed Sci. Technol. 166-167, 113-121. https://doi.org/10.1016/j.anifeedsci.2011.04.060 (2011).

35. AOAC. Official Methods of Analysis 15th edn. (IAOAC, Zürich, 1990).

36. AFNOR. Starch Determination Polarimetric Method. French Association for Standardization, 2nd edn, 123-125 (AFNOR editions, 1985).

37. Rohart, F., Gautier, B., Singh, A. \& Lê Cao, K.-A. mixOmics: an R package for 'omics feature selection and multiple data integration. PLoS Comput. Biol. 13, e1005752. https://doi.org/10.1371/journal.pcbi.1005752 (2017).

38. Wallace, R. et al. The rumen microbial metagenome associated with high methane production in cattle. BMC Genom. 16, 839. https://doi.org/10.1186/s12864-015-2032-0 (2015).

39. Guitton, Y. et al. Create, run, share, publish, and reference your LC-MS, FIA-MS, GC-MS, and NMR data analysis workflows with the Workflow4Metabolomics 3.0 Galaxy online infrastructure for metabolomics. Int. J. Biochem. Cell Biol. 93, 89-101. https://doi. org/10.1016/j.biocel.2017.07.002 (2017).

40. van der Kloet, F. M., Bobeldijk, I., Verheij, E. R. \& Jellema, R. H. Analytical error reduction using single point calibration for accurate and precise metabolomic phenotyping. J. Proteome Res. 8, 5132-5141. https://doi.org/10.1021/pr900499r (2009).

41. De Barbieri, I. et al. Programming rumen bacterial communities in newborn Merino lambs. Small Rumin. Res. 129, 48-59. https ://doi.org/10.1016/j.smallrumres.2015.05.015 (2015).

42. Romero-Pérez, A. et al. Effects of 3-nitrooxypropanol and monensin on methane production using a forage-based diet in Rusitec fermenters. Anim. Feed Sci. Technol. 220, 67-72. https://doi.org/10.1016/j.anifeedsci.2016.07.013 (2016).

43. Weimer, P. J. Redundancy, resilience and host specificity of the ruminal microbiota: implications for engineering improved ruminal fermentations. Front. Microbiol. 6, 296. https://doi.org/10.3389/fmicb.2015.00296 (2015).

44. Meale, S. J. et al. Development of ruminal and fecal microbiomes are affected by weaning but not weaning strategy in dairy calves. Front. Microbiol. 7, 582. https://doi.org/10.3389/fmicb.2016.00582 (2016).

45. Meale, S. J. et al. Weaning age influences the severity of gastrointestinal microbiome shifts in dairy calves. Sci. Rep. 7, 198. https:// doi.org/10.1038/s41598-017-00223-7 (2017).

46. Beauchemin, K. A., Ungerfeld, E. M., Eckard, R. J. \& Wang, M. Review: Fifty years of research on rumen methanogenesis: lessons learned and future challenges for mitigation. Animal 14, S2-S16. https://doi.org/10.1017/s1751731119003100 (2020).

47. Haisan, J. et al. The effects of feeding 3-nitrooxypropanol on methane emissions and productivity of Holstein cows in mid lactation. J. Dairy Sci. 97, 3110-3119. https://doi.org/10.3168/jds.2013-7834 (2014).

48. Reynolds, C. K. et al. Effects of 3-nitrooxypropanol on methane emission, digestion, and energy and nitrogen balance of lactating dairy cows. J. Dairy Sci. 97, 3777-3789. https://doi.org/10.3168/jds.2013-7397 (2014).

49. Lopes, J. C. et al. Effect of 3-nitrooxypropanol on methane and hydrogen emissions, methane isotopic signature, and ruminal fermentation in dairy cows. J. Dairy Sci. 99, 5335-5344. https://doi.org/10.3168/jds.2015-10832 (2016).

50. Lateur-Rowet, H. J. \& Breukink, H. J. The failure of the oesophageal groove reflex, when fluids are given with an oesophageal feeder to newborn and young calves. Vet. Q. 5, 68-74. https://doi.org/10.1080/01652176.1983.9693874 (1983).

51. Orskov, E. R. \& Benzie, D. Studies on the oesophageal groove reflex in sheep and on the potential use of the groove to prevent the fermentation of food in the rumen. Br. J. Nutr. 23, 415-420. https://doi.org/10.1079/bin19690048 (1969).

52. Cunha, C. S. et al. Assessing the impact of rumen microbial communities on methane emissions and production traits in Holstein cows in a tropical climate. Syst. Appl. Microbiol. 40, 492-499. https://doi.org/10.1016/j.syapm.2017.07.008 (2017).

53. Rychlik, J. L. \& May, T. The effect of a methanogen, Methanobrevibacter smithii, on the growth rate, organic acid production, and specific ATP activity of three predominant ruminal cellulolytic bacteria. Curr. Microbiol. 40, 176-180. https://doi.org/10.1007/ s002849910035 (2000).

54. Ramayo-Caldas, Y. et al. Identification of rumen microbial biomarkers linked to methane emission in Holstein dairy cows. J. Anim. Breed. Genet. 137, 49-59. https://doi.org/10.1111/jbg.12427 (2020).

55. Kittelmann, S. et al. Two different bacterial community types are linked with the low-methane emission trait in sheep. PLoS ONE 9, e103171. https://doi.org/10.1371/journal.pone.0103171 (2014). 
56. Vyas, D. et al. The combined effects of supplementing monensin and 3-nitrooxypropanol on methane emissions, growth rate, and feed conversion efficiency in beef cattle fed high-forage and high-grain diets. J. Anim. Sci. 96, 2923-2938. https://doi.org/10.1093/ jas/sky174 (2018).

57. Martinez-Fernandez, G. et al. 3-NOP vs. halogenated compound: methane production, ruminal fermentation and microbial community response in forage fed cattle. Front. Microbiol. 9, 1582. https://doi.org/10.3389/fmicb.2018.01582 (2018).

58. Danielsson, R., Schnürer, A., Arthurson, V. \& Bertilsson, J. Methanogenic population and $\mathrm{CH}_{4}$ production in Swedish dairy cows fed different levels of forage. Appl. Environ. Microbiol. 78, 6172-6179. https://doi.org/10.1128/AEM.00675-12 (2012).

59. Morgavi, D. P., Martin, C., Jouany, J. P. \& Ranilla, M. J. Rumen protozoa and methanogenesis: not a simple cause-effect relationship. Br. J. Nutr. 107, 388-397. https://doi.org/10.1017/S0007114511002935 (2012).

60. Zhou, Z., Meng, Q. \& Yu, Z. Effects of methanogenic inhibitors on methane production and abundances of methanogens and cellulolytic bacteria in in vitro ruminal cultures. Appl. Environ. Microbiol. 77, 2634-2639. https://doi.org/10.1128/aem.02779-10 (2011).

61. Wallace, R. J. et al. Archaeal abundance in post-mortem ruminal digesta may help predict methane emissions from beef cattle. Sci. Rep. 4, 5892. https://doi.org/10.1038/srep05892 (2014).

62. Romero-Pérez, A. et al. Effects of 3-nitrooxypropanol on methane production using the rumen simulation technique (Rusitec). Anim. Feed Sci. Technol. 209, 98-109. https://doi.org/10.1016/j.anifeedsci.2015.09.002 (2015).

63. Morgavi, D. P., Forano, E., Martin, C. \& Newbold, C. J. Microbial ecosystem and methanogenesis in ruminants. Animal 4, 10241036. https://doi.org/10.1017/S1751731110000546 (2010)

64. Bauchop, T. \& Mountfort, D. O. Cellulose fermentation by a rumen anaerobic fungus in both the absence and the presence of rumen methanogens. Appl. Environ. Microbiol. 42, 1103-1110. https://doi.org/10.1128/Aem.42.6.1103-1110.1981 (1981).

65. Hess, M. et al. Anaerobic fungi: past, present, and future. Front. Microbiol. https://doi.org/10.3389/fmicb.2020.584893 (2020).

\section{Acknowledgements}

SM received funding from the European Union's Horizon 2020 research and innovation program under the Marie Sklodowska-Curie Grant Agreement \#658126. CS received a fellowship from the Fundación Alfonso Martín Escudero. This research was partially funded by the French National Research Agency (ANR) through the FACCE-JPI project RumenStability. The authors wish to thank Florence Fournier and personnel at the Herbipole for animal care, and Dominique Graviou for laboratory technical support. We are grateful to the INRAE MIGALE bioinformatics platform (http://migale.jouy.inra.fr) for providing computational resources.

\section{Author contributions}

S.M., D.M., conceived the study. S.M., C.S., C.M., S.D., and D.M. made substantial contributions to the design of the work. S.M., M.P., A.B. performed bioinformatics analysis. H.B., M.L. performed metabololomic analysis. All authors were involved in the interpretation of data. S.M. drafted the manuscript that was critically revised by M.P., S.D., D.Y.R., and D.M. All authors reviewed and approved the manuscript.

\section{Competing interests}

SD declared he was employed by DSM Nutritional Products, which is the owner and patented 3-NOP.

\section{Additional information}

Supplementary Information The online version contains supplementary material availlable at https://doi. org/10.1038/s41598-021-82084-9.

Correspondence and requests for materials should be addressed to D.P.M.

Reprints and permissions information is available at www.nature.com/reprints.

Publisher's note Springer Nature remains neutral with regard to jurisdictional claims in published maps and institutional affiliations.

(c) (i) Open Access This article is licensed under a Creative Commons Attribution 4.0 International License, which permits use, sharing, adaptation, distribution and reproduction in any medium or format, as long as you give appropriate credit to the original author(s) and the source, provide a link to the Creative Commons licence, and indicate if changes were made. The images or other third party material in this article are included in the article's Creative Commons licence, unless indicated otherwise in a credit line to the material. If material is not included in the article's Creative Commons licence and your intended use is not permitted by statutory regulation or exceeds the permitted use, you will need to obtain permission directly from the copyright holder. To view a copy of this licence, visit http://creativecommons.org/licenses/by/4.0/.

(C) The Author(s) 2021 